\title{
Open Data Visualization in Danish Schools: A Case Study
}

\author{
Mubashrah Saddiqa \\ Department of Electronic Systems \\ Aalborg University, Aalborg \\ Denmark \\ mus@es.aau.dk \\ Lise L. Rasmussen
Department of Communication \\ Aalborg University, Copenhagen \\ Denmark \\ liselykke@hum.aau.dk \\ Birger Larsen \\ Department of Communication \\ Aalborg University, Copenhagen \\ Denmark \\ birger@hum.aau.dk \\ Rikke Magnussen \\ Department of Communication \\ Aalborg University, Copenhagen \\ Denmark \\ rikkem@hum.aau.dk

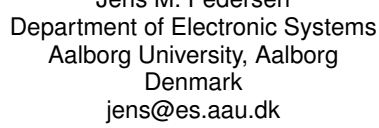

\begin{abstract}
Increasingly public bodies and organizations are publishing Open Data for citizens to improve their quality of life and solving public problems. But having Open Data available is not enough. Public engagement is also important for successful Open Data initiatives. There is an increasing demand for strategies to actively involve the public exploiting Open Data, where not only the citizens but also school pupils and young people are able to explore, understand and extract useful information from the data, grasp the meaning of the information, and to visually represent findings. In this research paper, we investigate how we can equip our younger generation with the essential future skills using Open Data as part of their learning activities in public schools. We present the results of a survey among Danish school teachers and pupils. The survey focuses on how we can introduce Open Data visualizations in schools, and what are the possible benefits and challenges for pupils and teachers to use Open Data in their everyday teaching environment. We briefly review Copenhagen city's Open Data and existing open source software suitable for visualization, to study which open source software pupils can easily adapt to visualize Open Data and which data-sets teachers can relate to their teaching themes. Our study shows that introducing Open Data visualizations in schools make everyday teaching interesting and help improving pupils learning skills and that to actively use Open Data visualizations in schools, teachers and pupils need to boost their digital skills.
\end{abstract}

\section{Keywords}

Open data, visualization's tools, interactive visualizations, educational resource, pupils.

\section{INTRODUCTION}

Open Data is freely available data for anyone to use, share and re-publish it anywhere and for any purpose. Mostly, Open Data is published by governments, public sectors, researchers and organizations such as data about transport, budgets, business, environment, maps, science, products, education, sustainability, legislation, libraries, economics, culture, development, design and finance. Open Data has the power to revolutionize and disrupt the way societies are governed. Being used in different sectors on a wider scale, there are many examples of how Open Data can save lives and change the way we live and work [1]. Organizations in many countries are beginning to publish large data-sets which are open for the public. But, the availability of Open Data alone is not enough to ensure that it is made use

Permission to make digital or hard copies of all or part of this work for personal or classroom use is granted without fee provided that copies are not made or distributed for profit or commercial advantage and that copies bear this notice and the full citation on the first page. To copy otherwise, or republish, to post on servers or to redistribute to lists, requires prior specific permission and/or a fee. and bring useful results. Without some form of aggregation, it can be hard for users to make sense of Open Data and understand it if they have no or little experience of processing and data analysis. Visualizations play an essential role as they are an effective way of interacting with large amounts of data from different fields, ranging from history [2] to economics [3] to basic science [4]. It provides a powerful means both to make sense of data and to present what has been discovered. With the advent of new interactive visualization techniques, presentation of data in a pictorial or graphical format makes it easier for a layman to understand large data-sets [5]. Presently, visualizations are widely used in news, books, internet, health, and economics [6] and having a limited knowledge of visualization can be a serious handicap.

As, society as a whole, is becoming increasingly digitized, it becomes essential to develop new research and educational models in schools to improve data-based digital competencies among students [7]. Many European countries have acknowledged the potential of digital competencies [8], and several have taken steps to introduce Open Data in schools and have started Open Data projects, e.g., Open Data for education competition in Northern Ireland with the intentions to use ex- 
isting Open Data to assist teaching in primary and secondary schools ${ }^{1}$.

The concept of Open Data can be made more interesting for school children and pupils by introducing visualizations and they may understand its potential and respond more quickly when exposed to visualization techniques. Therefore, to optimize the engagement of the young generation with Open Data, we believe that they must be familiarized with visualization tools, (e.g. charts, geographical maps and other types of representations) at an elementary level [9]. These simple visualizations become more powerful if they are created in relation to Open Data from domains that are of importance to the pupils, e.g. using data from their own city to detect and understand problems that are pertinent to their local area and everyday life [10]. With the availability of Open Data, new opportunities arise for all kinds of organizations, including government agencies, not-for-profits and businesses and allows them to come up with new ways of addressing problems in society. These include predictive health care [11], improving the transport system [12], [13] and transparency [14]. Open Data also opens up a wide variety of mostly unexploited possibilities of their use in education, e.g. in the form of visualizations. Although attention on visualization literacy [15], [16] continues to grow in different disciplines [17] there is still an ongoing discussion of the relative merits of different visualization platforms [18], [19]. Several studies [20], [21] illustrate people's limited knowledge in understanding data visualizations, indicating the urgency to address the problem. Recent studies [22], [23] pointed towards defining tactics to value visualization literacy, while others addressed how to improve it, using basic pedagogic ideas. Although many new teaching models [24], [25] have been presented with the purpose of increasing visualization literacy among the public, but not much attention paid on the ground level, i.e. schools. Open Data visualizations [26] based on local information can not only help to make visualization literacy easy and interesting but can also improve civic awareness and learning behaviors among pupils. Large and complex open data-sets can be further simplified and visualized for use in the teaching of, e.g. basic physics, mathematics and statistical methods, geography, social science and data handling in general. These data-sets not only make the teaching interesting and interactive but also develop skills among pupils to understand and ask questions about different facts of their local areas.

In this research work we reflect on the importance of Open Data visualizations on educational aspects, e.g. how Open Data visualizations can best facilitate education, what are the required skills to work with Open

\footnotetext{
${ }^{1}$ https://www.europeandataportal.eu/en/ highlights/open-data-schools
}

Data and its presentations at school level, and what would be the constraints and problems when working with Open Data visualizations at a basic school level? We address the following research questions:

- What would be the benefits of introducing Open Data and its visualization in the teaching tasks?

- How can Open Data visualizations be used in education especially at the school level?

- How can visualization facilitate understanding of Open Data in the educational domain?

- Which tools are considered user-friendly and effective in visualizing Open Data in schools?

We present results from a survey addressed to pupils and teachers to investigate these research questions. We briefly review the Open Data of the City of Copenhagen through its Open Data platforms. To visualize Open Data at a school level, we also analyzed userfriendliness of existing visualization technologies. We visualize concrete open data-sets of Copenhagen using existing visualization tools and presented the results to school pupils and teachers. These specific Open Data visualization examples are used during the interviews to provide a reference on how teachers could relate to them as part of their teaching. Their feedback is used to identify the interesting open data-sets, challenges and problems that need to be tackled in order to work with Open Data visualizations in the schools.

The paper is organized as follows: Section 2 describes the motivation and methodology. Section 3 presents the analysis of the open data-sets of the City of Copenhagen with some suggested educational open data-sets corresponding to specific educational domains and a short review of existing visualization techniques. Section 4 , describes the setup and results of the pupils-teachers survey. The conclusion of the study is given in section 5 .

\section{MOTIVATION AND METHODOL- OGY}

There is an increased number of governments and companies using Open Data to offer new services and products to the citizen, which signaled Open Data as one of the building blocks for innovation. Using Open Data effectively could help to save hours of unnecessary waiting time on the roads and help to reduce energy consumption. Using Open Data, newly developed mobile applications aim to make our lives a little easier and by using these applications we could have access to realtime information to minimize travel time, e.g. MinRejseplan ${ }^{2}$ mobile application in Denmark. To equip

\footnotetext{
2 https://www. nordjyllandstrafikselskab.dk/
} Billetter---priser/MinRejseplan 
our younger generation with the required digital skills for future challenges, it is important that teachers are aware of and able to use Open Data as an educational resource that allows them to develop data and digital skills among younger generation of pupils.

Denmark is one of the leading countries with the most up-to-date Open Data. Denmark has a national platform ${ }^{3}$ for the cities to publish Open Data, providing a common entry point for accessing Open Data. According to the Digital Economy and Society Index (DESI) 2018, Denmark is among the most digital countries in Europe $^{4}$. The report documents that 94 percent of the country's public facilities and services are online and are highly advanced. Copenhagen, the capital of Denmark, is also famous for its smart city initiatives. The city has a large collection of Open Data available for its citizens. But, the social impact of Open Data is very limited due to the lack of public awareness according to Open Data Maturity report 2018 [27]. Therefore data literacy becomes imperative for the citizen of Denmark especially for the future generation, in order to make use and contribute actively for the improvement of digital services. There is a growing demand to take initiatives that aim at advancing digital skills and creating new interactive learning and teaching resources at the school level. Open Data is an open resource that can facilitates teaching with real information and allows pupils to develop data and digital skills. In this research work, we focus in particular on how to create understanding and representation of Open Data as an educational resource for public school pupils. We focus in particular on the city's many types of data and how to put them into use especially in an educational context. For example, there may be data about traffic, pollution, light and the use of different areas and facilities. These data-sets can easily relate to different subjects, such as Science, Mathematics or Geography subjects. At the same time, it is possible to compare this data with other areas of the city.

To understand how Open Data visualizations facilitate educational activities we conducted a survey with Danish public schools in Copenhagen. The survey is conducted from August 2018 to December 2018. Six Danish public schools, 10 school teachers and 21 school pupils of 7th grades aged between 13-14 years participated in the survey. The survey is formulated over interviews, questionnaire, and observations. The detailed methodology is discussed in Section 4 below. To investigate the research questions through our survey, we fur-

\footnotetext{
3 http://www.opendata.dk/

${ }^{4}$ http://ec.europa.eu/information_society/ newsroom/image/document/2018-20/ dk-desi_2018-country-profile_eng_ B43FFE87-A06F-13B2-F83FA1414BC85328_ $52220 . p d f$
}

ther sub-divided the research questions into three categories.

\section{The Data Perspective:}

- What would be the benefits of introducing Open Data in the teaching tasks?

- What types of data are already used in the schools?

- What would be the most interesting open data-sets?

- What benefits do teachers think Open Data visualizations could have?

\section{Teachers/Pupils Competencies Perspective:}

- Which skills and competencies do pupils and teachers already have in working with data and presenting them?

- Which skills and competencies would be necessary for pupils and teachers to work and present Open Data?

\section{The Visualization's tool Perspective:}

- Which visualization tools are appropriate and easy to adapt for school teachers and pupils?

- Which visualization tools do teachers already use in their teaching?

- What are the possible challenges in adopting new visualization tools, e.g. language barriers or installation of the systems?

To identify perspective on teachers/pupils skills and competencies, we collaborate with Danish public school teachers and pupils to learn their views about bringing Open Data into their classrooms and to identify skills they already have. The details are discussed in Section 4 below. To investigate perspectives about data and visualization tools, we reviewed and analyzed the open data-sets of the City of Copenhagen and identified which domains of Open Data could be used in the different domain of education, e.g. Mathematics, Science, and Geography. We also make a comparison of different available user-friendly visualization's tools to find out which of them could be the best option for school teacher and pupils or if there is a need to develop Open Data visualization interface specifically for schools.

In the next section, we will briefly review open data-sets of Copenhagen to discover possible data-sets which can be used as part of different subjects in schools and contains interesting information about the city and its local areas. And we analyze user-friendly visualization's tools to identify those we could adapt for the rest of the survey. 


\section{ANALYSIS OF OPEN DATA-SETS AND USER-FRIENDLY TECHNOLO- GIES}

In this section, we first analyze the open data-sets of Copenhagen city to categorized Open Data themes corresponding to different educational domain, e.g. Science, Mathematics, Geography and Social science. In order to utilize the Open Data in education, we also focus on the availability of the data-sets, data formats and data types. Next for visualization of Open Data, we analyze existing user-friendly visualization's tools and discuss their main features.

\subsection{Open Data-sets of Copenhagen}

The Copenhagen Open Data website ${ }^{5}$ has a large number of data-sets from all over the City. There are datasets, for example on traffic, parking, the city's physical infrastructure, as well as data on population, culture and education. More than 280 data-sets are available in different formats and can be found in the Copenhagen city website. In order to make use of Open Data using visualizations at school level, it is important to understand the different formats and characteristics of datasets. The Open Data of Copenhagen city can be categorized into ten general themes. These general themes of Open Data with corresponding sub-domains are listed in Table.1. The data sets includes static data, dynamic

\begin{tabular}{|l|l|}
\hline \multicolumn{1}{|c|}{ General Themes } & \multicolumn{1}{|c|}{ Sub-domain } \\
\hline Environment & $\begin{array}{l}\text { Nature, Water Quality, Air } \\
\text { Quality, Pollution, etc. }\end{array}$ \\
\hline Governance & $\begin{array}{l}\text { Demographics, Elections, } \\
\text { Census, Transparency, etc. }\end{array}$ \\
\hline Health \& Care & $\begin{array}{l}\text { Social care, Care Homes, } \\
\text { Child Care, etc. }\end{array}$ \\
\hline Infrastructure & $\begin{array}{l}\text { Roads, Buildings, Locations, } \\
\text { Planning, etc. }\end{array}$ \\
\hline Transport & $\begin{array}{l}\text { Traffic, Parking, Public Trans- } \\
\text { port, Pedestrian, Cyclist, etc. }\end{array}$ \\
\hline Community & $\begin{array}{l}\text { Society, Housing, Employ- } \\
\text { ment, etc. }\end{array}$ \\
\hline Education & Schools, Kindergartens, etc. \\
\hline Energy & $\begin{array}{l}\text { Solar Energy, Consumption, } \\
\text { Carbon Emission, etc. }\end{array}$ \\
\hline Culture \& Sports & $\begin{array}{l}\text { Entertainment, Tourism, Cul- } \\
\text { tural Locations, etc. }\end{array}$ \\
\hline Economy & Finance, Economy, etc. \\
\hline
\end{tabular}

Table 1: General Themes of Copenhagen Open Data

data, geographical and live data in different formats. In Table.2, the main characteristics of the Open Data of Copenhagen city are presented. Most of the datasets are up-to-date and timely processed. The statistical contribution of major themes of Copenhagen city is

$5 \overline{\text { https://data.kk.dk/ }}$

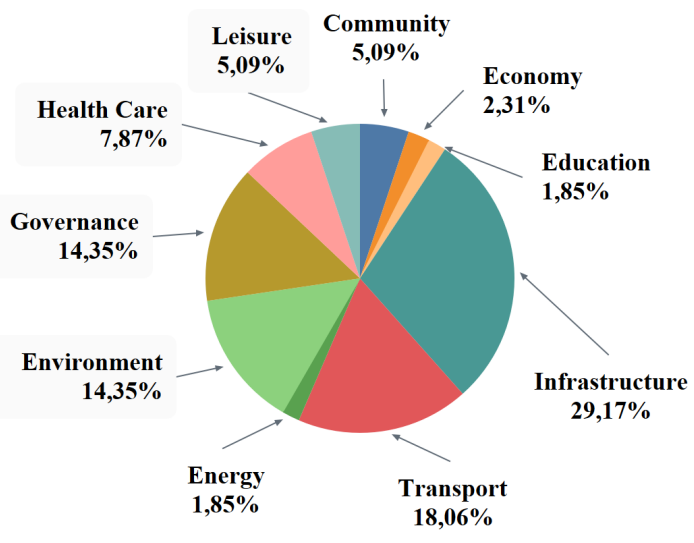

Figure 1: Statistical view of Open Data Major Themes

shown in Figure.1. This brief analysis will enable us to identify some of the relevant data categories which will be used as part of specific educational domains. The Open Data review of city enabled us to explore 4 impact domains (educational themes) discussed in [28] with associated sub-domains corresponding to main educational domains, i.e. Mathematics, Science and Geography. Figure.2, represents the possible mapping of

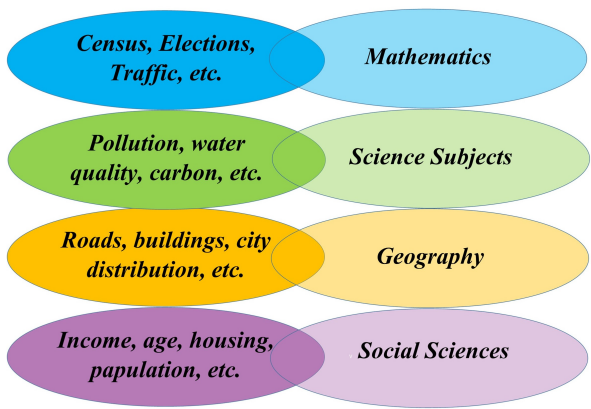

Transport and $\bigcirc$ Environment $\bigcirc$ Infrastructure Demography
Governance

Figure 2: Example of Open Data themes corresponding to educational domains

Open Data educational themes to the educational sector. Table.3, presents different sub-domains of Open Data (educational themes) and examples of their possible use corresponding to specific educational domains. This analysis will enable us to ask teachers which of the different available data-sets and formats can be used during teaching tasks, which data-sets are interesting and how they could be used as an aid to make teaching tasks more interactive.

\subsection{Review of User-Friendly Visualization Tools}

A large number of data visualization technologies have been developed over the last decade to support the ex- 


\begin{tabular}{|l|l|l|c|}
\hline \multicolumn{1}{|c|}{ General Themes } & \multicolumn{1}{|c|}{ Data Types } & \multicolumn{1}{c|}{ Format } & $\begin{array}{c}\text { Data- } \\
\text { sets }\end{array}$ \\
\hline Environment & Live/Sensors, Multi-dimensional & CSV, EXCEL & 33 \\
\hline Governance & Statistical, Multi-dimensional & CSV, EXCEL, SHP(Shapefile) & 22 \\
\hline Health and Care & Statistical & CSV, EXCEL & 17 \\
\hline Infrastructure & Geographical, Live/Sensors, Statistical & CSV, EXCEL, DWG(Drawing) & 63 \\
\hline Transport & Live/Sensors, Statistical & CSV, EXCEL, KLM, GeoJson & 39 \\
\hline Community & Statistical, Historical & CSV, EXCEL & 29 \\
\hline Education & Statistical & CSV, EXCEL, GeoJson & 5 \\
\hline Energy & Live/Sensors, Statistical & CSV, EXCEL, KLM & 4 \\
\hline Culture \& Sports & Statistical, Historical & CSV, EXCEL & 9 \\
\hline Economy & Statistical, Historical & CSV, EXCEL & 9 \\
\hline
\end{tabular}

Table 2: Characteristics of General Themes of Copenhagen Open Data

\begin{tabular}{|l|l|l|}
\hline Educational Domain & Educational Themes & Examples \\
\hline Sciences Subjects & $\begin{array}{l}\text { Environmental Data: Pollution, water } \\
\text { quality, traffic, carbon Level, energy, etc. }\end{array}$ & $\begin{array}{l}\text { To view the city's pollution within } \\
\text { the city, e.g. carbon level. }\end{array}$ \\
\hline Mathematics & $\begin{array}{l}\text { Statistical Data: Gender, population, age, } \\
\text { housing, education, etc. }\end{array}$ & $\begin{array}{l}\text { To make comparison of different } \\
\text { details, e.g. gender comparison. }\end{array}$ \\
\hline Geography & $\begin{array}{l}\text { Geographic Data: City distribution, Build- } \\
\text { ings, Roads, Locations, etc. }\end{array}$ & $\begin{array}{l}\text { To view the locations of buildings, } \\
\text { roads and areas etc. }\end{array}$ \\
\hline Social Science & $\begin{array}{l}\text { Demographic Data: Education, national- } \\
\text { ity, income, work, culture, etc. }\end{array}$ & $\begin{array}{l}\text { To view the details about popula- } \\
\text { tion, age, income, employment and } \\
\text { education etc. }\end{array}$ \\
\hline
\end{tabular}

Table 3: Open Data main themes and corresponding educational domain

ploration of large data-sets. In order to interact with Open Data, it is important to visualize it. For this, visualization technologies and software are required, which are user-friendly, needs no programming knowledge, and are supposed to be simple in use if used by the school teachers and pupils. According to The Tech Terms Computer Dictionary ${ }^{6}$, user-friendly means a software interface that is easy to learn and easy to use. It should be simple with easy access to different tools and options, and with minimal explanation for how to use them. For school pupils, We are interested in tools which are

- user-friendly

- needs no coding/programming requirements and

- support the most common formats, (e.g. CSV, Excel, Google sheets etc.)

- provide a platform where teachers and pupils can share their visualizations

- provide a public forum for inspiration, collaboration with others and share experiences

- provide a free license for pupils and teachers

\footnotetext{
6 https://techterms.com/definition/ user-friendly
}

We analyzed six different tools which are often used for visualization purposes. In the next sections, we discussed their main features and compare them to one another based on the criteria defined above to find the best possible tools adapted for visualization at school levels. The comparison is given in Table.4.

\section{Tableau}

Tableau $^{7}$ is a data visualization tool and provides different products with a one-year free license under its academic program. The academic suite includes:

- Tableau Desktop

- Tableau Prep

- Tableau Online

- Tableau public to collaborate with others visualizations and experiences

- Customer support and community forum

Tableau Desktop is used to connect with various types of data and for creating visualizations. Tableau Prep transforms data for analysis and allows users to combine and clean data prior to visualization. With Tableau Online pupils can easily share and collaborate their work by uploading workbooks to a site managed by instructors. Sites are issued for 1 year and allow for 100

$7 \overline{\text { https://www }}$ tableau.com/ 


\begin{tabular}{|l|c|c|c|c|c|c|}
\hline \multicolumn{1}{|c|}{ Tools } & $\begin{array}{c}\text { Free } \\
\text { License }\end{array}$ & $\begin{array}{c}\text { Academic } \\
\text { Program }\end{array}$ & $\begin{array}{c}\text { No } \\
\text { Programming }\end{array}$ & $\begin{array}{c}\text { Teachers' } \\
\text { Site }\end{array}$ & $\begin{array}{c}\text { User } \\
\text { Friendly }\end{array}$ & $\begin{array}{c}\text { Public } \\
\text { Forum }\end{array}$ \\
\hline Tableau & $\mathrm{x}$ & $\mathrm{x}$ & $\mathrm{x}$ & $\mathrm{x}$ & $\mathrm{x}$ & $\mathrm{x}$ \\
\hline QlikView & $\mathrm{x}$ & $\mathrm{x}$ & $\mathrm{x}$ & & $\mathrm{x}$ & $\mathrm{x}$ \\
\hline Power BI & $\mathrm{x}$ & & $\mathrm{x}$ & & $\mathrm{x}$ & $\mathrm{x}$ \\
\hline Excel & $\mathrm{x}$ & & $\mathrm{x}$ & & $\mathrm{x}$ & $\mathrm{x}$ \\
\hline Datawrapper & $\mathrm{x}$ & & $\mathrm{x}$ & & $\mathrm{x}$ & $\mathrm{x}$ \\
\hline Google Maps & $\mathrm{x}$ & & $\mathrm{x}$ & & $\mathrm{x}$ & $\mathrm{x}$ \\
\hline
\end{tabular}

Table 4: Comparison of commonly used open source software based on defined criteria

simultaneous users. There is no need for any coding knowledge to work with Tableau.

\section{QlikView}

QlikView $^{8}$ is a user-friendly, interactive open source visualization tool with no requirements of coding knowledge. Visualizations can be shared with up to 5 users. It is compatible with default and custom data connectors and also import data from popular databases. Qlik also provides an academic package for students and teachers which includes the following resources:

- Qlik Software

- Qlik Continuous Classroom online learning platform

- Data Analytic curriculum featuring lecture notes, on-demand videos, handouts, activities and realworld, interactive business use cases

- Qlik Community Academic Program Space, a forum for professors and students to access resources, collaborate with others and share experiences and Qlik Customer Support

\section{Power BI}

Power $\mathrm{BI}^{9}$ is a collection of software services, apps, and connectors that work together to turn data into interactive visualizations with out any programming knowledge. Power BI easily connect to different data sources. Pupils can get a free limited version of Power BI but not an academic program with full resources. BI also provides a public forum to collaborate with others but it does not provide a separate free site manged by instructors to collaborate with their pupils.

\section{Excel}

Excel ${ }^{10}$ is part of the well-known Microsoft Office suite and also supports data analysis. One of the greatest advantages of Excel is its flexibility, as it puts little or no constraints on the user's ability to create visualizations.

\footnotetext{
${ }^{8}$ https://www.qlik.com/us/products/qlikview

${ }^{9}$ https://powerbi.microsoft.com/en-us/

10 office.microsoft.com/excel
}

Excel does not provides specific academic programs. No programming knowledge is required for visualization purposes. Excel also support commonly used formats, (CSV, Excel and sheets etc.). Excel provides low barriers and sufficient tooling, but it stops at a medium data level.

\section{Datawrapper}

Datawrapper $^{11}$ is a user friendly and mobile-friendly data visualization tool. No programming is required for visualizing data. It supports 10,000 monthly charts to publish. It is compatible with CSV, Excel and Google sheets and provides a community forum to share experiences and problems with others but there is no academic program for pupils or teachers.

\section{Google Maps}

We also list Google Maps as a visualization tool because geographical data can easily be visualized using Google Maps. In addition, it is a familiar tool for pupils and teachers and mostly are already familiar with many of its features, e.g. from planing travels, finding locations and receiving traffic information. It is also userfriendly and requires no programming skills.

We selected Tableau and Google Maps as the visualization tools, which we used as examples during the interviews. Tableau fulfills the required criteria and Google Maps is a good option as most of the people already familiar with its features and functions.

\section{PUPILS-TEACHERS SURVEY}

In order to identify the teacher's perspective on the role that Open Data visualization can play for school pupils, we surveyed Danish public school teachers and pupils. The survey includes interviews with teachers and a twoday pilot test with school pupils and teachers.

\subsection{Participants}

For teachers' study, we recruited 10 teachers from 6 different Danish public schools. The teachers were identified according to their subject, experience and age. The

\footnotetext{
$11_{\text {https }}$ ///www. datawrapper.de/
} 
focus subjects were Mathematics, Science and Geography. The teachers have teaching experience from 3 years to 15 years in the above-mentioned subjects and were aged between 25-45 years. For pupils' study, we ran a pilot test for two days in one of the Danish public school. Two (science and maths) teachers along with 21 students of 7th grade, aged between 13-14 years old participate in the pilot test.

\subsection{Setup}

Before the interviews, we delivered presentations of about 10 to 15 minutes on Open Data and its visualization using data-sets containing local information pertaining to the neighborhood around each school, and their possible usage at the school level as part of Mathematics, Science, or Geography. For instance, data-sets about the areal distribution of the city can provide interesting information for a Geography class. We transform bigger data-sets within different themes into smaller data-sets to present the local information relevant to respective school locations using Tableau and Google Maps. For example, Figure.3, represents Copenhagen

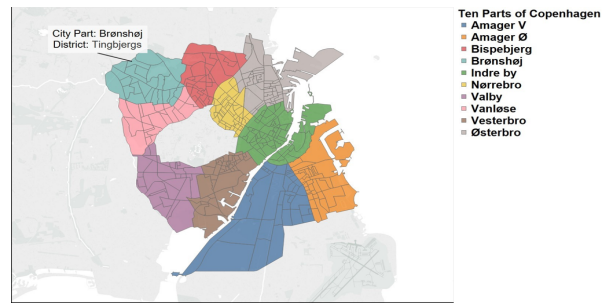

Figure 3: Areal distribution of Copenhagen

distribution into different parts, each with a unique color and further divided into smaller districts. It can be an interesting visualization for school pupils as they can locate their local areas easily. Figure.4 represents

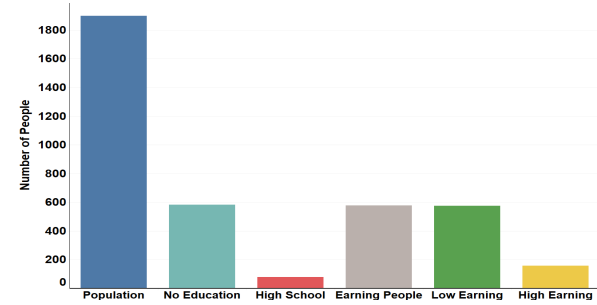

Figure 4: Statistical details of Tingbjerg

statistical details of Tingbjerg (district of Copenhagen) are presented, e.g population, education, low and high income. Using Google Maps, we visualize locations of the city's public schools according to their postal code numbers as a specific example for teachers and pupils. School pupils can use this map to get direction and measure the distance of their schools from their homes, different routes to their schools, more information about schools, e.g. schools website and address. These visualizations formulated over the local area information near the school, will help teachers to understand what type of data and information they could present to pupils during their teaching tasks as part of Mathematics, Science and other related subjects. For the pilot test with pupils, we requested the school administration to install the free academic package of Tableau on pupils and teachers computers. We provided teachers (math and science) beforehand with some specific visualizations which they could relate with their subjects, e.g. pollution level in different parts of the city at different times of the day, traffic passing through their areas at different parts of the day, the population details including age, education, and gender details. We also designed activities to explore the information through these specific visualizations, e.g. to find the carbon level of their area in morning and evening time, to find the gender details of their area etc. Pupils were also given a presentation, where we presented the visualizations of the data-sets from the educational Open Data themes given in Table.3, using Tableau.

\subsection{Procedure}

To investigate our research questions in different aspects discussed in Section 2, we conducted a teachers and pupils survey. The teachers' study was carried out with individual participants. Before the interviews, the participants were given a small introduction to Open Data and how they can adapt it as part of teaching tasks. We also asked both teachers and pupils for their consent to participate in the research work using video and audio devices. We used the Danish language for both teachers and pupils' study.

\subsubsection{Teacher's Study}

To achieve the best possible results and feedback on the three perspectives of our research questions, i.e.; the data perspective, the teachers perspective and the visualization tool perspective. We divide the teacher's study into three sessions, each session with 30 to 40 minutes. The overall time for teacher's study is 1.5 to 2 hours. To investigate the data perspective, in the first session, we presented short presentations of Open Data and its visualizations, existing open data-sets of Copenhagen according to the location of the schools and its possible usage at the school level as part of Mathematics, Science, and Geography subjects. To identify the visualization tools perspective, teachers will test the visualization tool Tableau, and try to visualize some of the provided ready to use open data-sets in the second session of the study. This will help us to identify which competencies teachers need when working with Open Data visualization and how convenient is it for the teachers to use. This is used as a background in order to investigate if they can use Tableau as part of their teaching. Teachers were provided with personal assistance to understand some main features of Tableau and Google Maps. In the third session, focus is given more on the 
teachers' perspective. We asked participants to fill out a questionnaire and answer several semi-structured questions which elicit their views about the following perspectives.

\section{Data perspective:}

We asked questions about data already in use and formats used for the presentation of data during teaching especially in Mathematics, Science, and Geography tasks. What is the pupils' level of understanding data in different grades?

\section{Teachers Perspective:}

How Open Data visualization facilitate education in schools? What will be the possible impact on their teaching environment and on the school pupils with the use of real examples using Open Data visualization. What are the teachers' views about the skills pupils need to work with Open Data and its presentation?

\section{Visualization tools Perspective:}

We investigate the adapting of visualization tool Tableau and Google Map in order to visualize Open Data in a simple way. Whether they encountered any technical issues or limitations of the visualization tool? What will be the possible challenges and problems?

In addition, we asked several semi-structured and optional open-ended questions about the problems, and their suggestion to successfully introduce Open Data visualization into the educational domain.

\subsubsection{Pilot Test}

The role of pupils are important to investigate how they work with data, what data-sets are interesting for pupils about their areas and how they interpret Open Data visualization. We ran a pilot test in a Danish public school with 7th grade pupils aged between 13-14 years old. The pupils worked with Open Data in their Physics/Chemistry and Mathematics lectures for two consecutive days. We presented a 5-10 minutes presentation about Open Data with the help of visualizations of data-sets within educational themes. These include air pollution, carbon level, traffic around their area and some population details of their area. After the presentation, teachers relate some of the visualizations as part of Chemistry, where they talked about air pollution and different gases responsible for the pollution. With the help of Open Data visualization, teacher pointed different areas where pollution level is higher than the other areas, e.g. train and central bus stations. Similarly, Maths' teacher also relates statistical data of the city to make comparisons. On the second-day, pupils were taught how to use Tableau. They were provided with small data-sets to visualize. All these activities were observed and documented using videos and still photos. The pilot test ended with a questionnaire in the form of simple questions. We asked pupils about their understanding with different types of data formats, e.g. Excel, spreadsheets, CSV and the types of graphs, e.g. line, bar, pie charts. Which type of graph do they understand easily? What are the interesting data-sets about their city? Based on the input from teachers and pupils we also analyzed the three main issues.

\subsection{Result}

In this section, we discuss outcomes from the pupilsteachers survey. The following are the more salient points from their feedback.

\section{Teachers/Pupils Perspective}

- Teachers are able to understand and describe the Copenhagen Open Data visualizations presented during the interviews.

- According to teachers describing the city using Open Data visualizations will bring new perspectives in school teaching and school pupils.

- Teachers already used tools like GeoGabra, Excel and Google Maps for basic data handling and presentations.

- Pupil are already familiar with different types of graphical representations of data in Mathematics and Science subjects, e.g. lines, bar and pie charts.

- The pupils are likely to learn more when working with actual data of their area using visualizations.

- The interactive visualization tools can help a lot in order to make things more clear and interesting for pupils, but they are relatively hard to use.

- Pupils needs both, the skills and the tools to actively work with Open Data as an educational resource.

- Teachers believe that pupils' involvement can be made more interactive during the class using real data and real examples.

\section{Data Perspective}

- The data cleaning and preparing concepts are not often used in teaching.

- Pupils use mostly data in tables to make graphs. It is not seen as difficult to introduce other formats of data in teaching, e.g. CSV and Excel formats.

- Pupils can use statistical Open Data of Copenhagen to understand mathematical methods, e.g. to make the comparison of different real-life facts of their local areas.

- The teachers agreed that they can introduce different formats of Open Data particularly the CSV in 4th7 th grades and Excel format from 5th-7th grades in mathematics subject. 


\section{Visualization's Tools Perspective}

- Teachers are comfortable with Tableau and Google Maps and believe that pupils can take benefits from these open source tools.

- Pupils from grade 4th-7th are also used to Google Maps from previous teaching experience. It will give more meaning if they can make use of it in their daily life using Open Data, e.g. they can use Open Data to locate play areas on Google maps to get directions and measure distances from their schools or homes.

- The Open Data visualization can play an important role in explaining more abstract things in a concrete way.

\subsection{Discussion}

In order to fully enable school pupils to understand and work with Open Data visualizations, introduction to data formats, data preparation, and data cleaning concepts need to be introduced as part of their subjects for example in Mathematics. Using Open Data visualization of different data-sets, e.g. traffic, population, economy, and education etc., of their city in subjects like Mathematics, Geography and Science class can give them a chance to work with actual data. For example, presenting pollution level near the school at different periods of the day not only makes Science class interesting but also improve civic awareness among younger generation as the simple local visualization allow them to discuss why the level is higher at some periods and how they can reduce it. Using interesting statistics about pupils age group in different cities could help Mathematics in understanding ratio and percentage problems with real data. Teachers are comfortable with Tableau as visualization tool because of its quite easy to use functionally without any coding and free licensing for pupils and teachers but they also point out some limitations, e.g. they need extra time and efforts to visualize related open data-sets. Google Maps is also seen as a good option for geographical representations and measuring distances or finding alternative routes and can be used as part of Geography class. Based on the survey, we also identified some of the challenges. It can be hard for public school teachers to directly use the Open Data available at city's Open Data portal. They will need smaller ready-to-use data-sets as part of their teaching subjects. Teachers need support and training workshops in order to use new visualization technologies at schools. The visualization tool Tableau and other open source tools are in English which could be a problem for public school pupils, but explaining some of the frequent functions in Danish can solve this problem. In order to bring Open Data in schools as an educational resource, our survey also suggests the development of a school friendly Open Data visualization interface that provides local real information in the form of interesting and simple visualization in pupils' native language as part of different subjects.

\section{CONCLUSION AND FUTURE WORK}

In this paper, we have presented a pupils-teachers survey as a first step, to study how Open Data visualization can be introduced in the schools to facilitate educational activities, which skills school teachers and pupils already have and which visualization tools could be used to visualize Open Data as part of teaching subjects. Our study includes the visualization of Copenhagen open data-sets, a review of existing user-friendly visualization techniques and software and a qualitative survey of teachers and pupils. Based on our survey, we concluded that Open Data visualization can be brought into schools as part of different teaching subjects especially Mathematics, Science, and Geography and it can plays an important role in improving the pupils learning behaviors, as teachers and pupils are able to relate real problems from different perspectives in everyday teaching. In order to use Open Data Visualization actively in different teaching subjects, teachers need to boost their digital skills, adopt new interactive learning and teaching resources as well as support the approaches for knowledge development. Insight from this study, advances the knowledge of our community about what are the skills needed for schools teachers to use the Open Data visualization in their teaching areas and how the availability and literacy of Open Data visualization in schools create opportunities for school pupils to come up with new ways of addressing and understanding society's problems.

As a future work, we are aiming to develop a schoolfriendly Open Data visualization interface that provides aid to teachers and pupils to visualize local area datasets within Open Data educational themes, compare these data-sets with other areas and suggests different activities for pupils as part of teaching tasks. We will first identify needs and requirements for school-friendly Open Data interface using the Requirements Engineering domain and then develop prototypes for testing and validation of the interface in the Danish public schools.

\section{REFERENCES}

[1] Dong, H., Singh, G., Attri, A., and El Saddik, A. Open data-set of seven Canadian cities. IEEE Access, Vol.5, pp.529-543, 2017.

[2] Friendly, M. A brief history of data visualization. In Handbook of data visualization, Springer, pp.15-56, 2008 
[3] Schwabish, J.A. An economist's guide to visualizing data. Journal of Economic Perspectives, Vol.28, No.1, pp.209-34, 2014.

[4] Jipeng, L., Fang, Y., and Yu, H. The Application of visualization in scientific computing technology to architectural design. International Forum on Computer Science-Technology and Applications, Vol.3, pp.93-96, 2009.

[5] Valkanova, N., et al. Public visualization displays of citizen data: design, impact and implications. International Journal of Human-Computer Studies, Vol.81, pp.4-16, 2015.

[6] Segel, E. and Heer, J. Narrative visualization: Telling stories with data. IEEE transactions on visualization and computer graphics, Vol.16, No.6, pp.1139-1148, 2010.

[7] Geoghegan-Quinn, M. Science 2.0: Europe can lead the next scientific transformation. In EuroScience open forum (ESOF), Keynote speech, 2014.

[8] Wastiau, P., et al. The use of ict in education: a survey of schools in europe. European Journal of Education, Vol.48, No.1, pp.11-27, 2013.

[9] Alper, B., et al. Visualization literacy at elementary school. In Proceedings of the 2017 CHI Conference on Human Factors in Computing Systems, ACM, pp.5485-5497, 2017.

[10] Atenas, J., and Havemann, L. Open data as open educational resources: case studies of emerging practice. Open Knowledge-Open Education Working Group, 2015.

[11] Ghassemi, M., Celi, L.A., and Stone, D.J. State of the art review: the data revolution in critical care. Critical Care, Vol.19, No.1, doi: http:// ccforum.com/content/19/1/118, 2015.

[12] Dawes, S.S., Vidiasova, L., and Parkhimovich, O. Planning and designing open government data programs: An ecosystem approach. Government Information Quarterly, Vol.33, No.1, pp.15-27, 2016 .

[13] Urry, J. Mobilities: new perspectives on transport and society. Routledge, 2016.

[14] Carlo Bertot, J., Jaeger, P.T., and Grimes, J.M. Promoting transparency and accountability through ICTs, social media, and collaborative e-government. Transforming government: people, process and policy, vol.6, No.1, pp.78-91, 2012.

[15] Abilock, D. Visual information literacy: Reading a documentary photograph. Knowledge Quest, Vol.36, No.3, pp.7-14, 2008.

[16] Taylor, C. New kinds of literacy, and the world of visual information. Literacy, 2003.

[17] Boy, J., Rensink, R.A., Bertini, E., and Fekete,
J.D. A principled way of assessing visualization literacy. IEEE transactions on visualization and computer graphics, Vol.20, No.2, pp.1963-1972, 2014.

[18] Kim, S-H., Boy, J., Lee, S., Yi, J.S. and Elmqvist, N. Towards an Open Visualization Literacy Testing Platform. IEEEVIS Workshop, 2014.

[19] Wolff, A., et al. Data literacy for learning analytics. In Proceedings of the Sixth International Conference on Learning Analytics and Knowledge, pp.500-501, ACM, 2016.

[20] Börner, K., Maltese, A., Balliet, R.N., and Heimlich, J. Investigating aspects of data visualization literacy using 20 information visualizations and 273 science museum visitors. Information Visualization, Vol.15, No.3, pp.198-213, 2016.

[21] Kennedy, H., Hill, R.L., Allen, W., and Kirk, A. Engaging with (big) data visualizations: Factors that affect engagement and resulting new definitions of effectiveness. First Monday, Vol.21, No.11, 2016.

[22] Boy, J., Rensink, R.A., Bertini, E., and Fekete, J.D. A principled way of assessing visualization literacy. IEEE transactions on visualization and computer graphics, Vol.20, No.12, pp.1963-1972, 2014.

[23] Lee, S., Kim, S.H., and Kwon, B.C. Vlat: Development of a visualization literacy assessment test. IEEE transactions on visualization and computer graphics, Vol.23, No.1, pp.551-560, 2017.

[24] Huron, S., Carpendale, S., Thudt, A., Tang, A., and Mauerer, M. Constructive visualization. In Proceedings of the 2014 Conference on Designing Interactive Systems, pp.433-442, ACM, 2014.

[25] Ruchikachorn, P., and Mueller, K. Learning visualizations by analogy: Promoting visual literacy through visualization morphing. IEEE transactions on visualization and computer graphics, Vol.21, No.9, pp.1028-1044, 2015.

[26] Graves, A., and Hendler, J. Visualization tools for open government data. In Proceedings of the 14th Annual International Conference on Digital Government Research, ACM, pp.136-145, 2013.

[27] Open Data Maturity in Europe 2018: New horizons for Open Data driven transformation. European data portal, 2018.

[28] Saddiqa, M., et al. Bringing Open Data into Danish schools and its potential impact on pupils. 15th International Symposium on Open Collaboration, ACM, 2019. 